\title{
Theoretical calculation and evaluation of neutron inducedreactions on $\mathrm{Pu}$ isotopes
}

\author{
Hairui Guo ${ }^{1, *}$, Yinlu $\mathrm{Han}^{2, * *}$, Tao Ye ${ }^{1,}$, Weili Sun ${ }^{1,}$, and Wendi Chen ${ }^{3}$, \\ ${ }^{1}$ Institute of Applied Physics and Computational Mathematics, Beijing 100094, China \\ ${ }^{2}$ China Institute of Atomic Energy, P.O. Box 275(41), Beijing 102413, China \\ ${ }^{3}$ Graduate School of China Academy of Engineering Physics, Beijing 100088, China
}

\begin{abstract}
The nuclear data on $\mathrm{n}+{ }^{239,240,242,244} \mathrm{Pu}$ reactions for the incident energy up to $200 \mathrm{MeV}$ are consistently calculated and evaluated in order to meet the design requirements of Generation-IV reactors and accelerator driven systems. The optical model, the distorted wave Born approximation theory, the Hauser-Feshbach theory, the fission model, the evaporation model, the exciton model and the intranuclear cascade model are used in the calculation, and new experimental data are taken into account. Our data are compared with experimental data and the evaluated data from JENDL-4/HE and TENDL. In addition, the variation tendency of reaction cross sections related to the target mass numbers is obtained, which is very important for the prediction of nuclear data on neutron-actinides reactions because the experimental data are lacking.
\end{abstract}

\section{Introduction}

Recent decades, Generation-IV reactors and accelerator driven systems (ADS) are under study to reduce the nuclear accident probability and minimize the production of nuclear waste. Pu isotopes are important fuel or radioactive waste, accurate nuclear data of neutron-induced reactions on Pu isotopes below $200 \mathrm{MeV}$ are needed to reduce uncertainties in the design and operation of these facilities.

At present, there are two nuclear data libraries including $\mathrm{n}+\mathrm{Pu}$ reaction data up to $200 \mathrm{MeV}$. One is JENDL4/HE library [1]. In this library, JENDL-4.0 is adopted at incident energies $E_{n} \leq 20 \mathrm{MeV}$ (the evaluation finished in 2009), and JENDL-HE2007 is adopted for $20 \leq E_{n} \leq 200$ $\mathrm{MeV}$, this evaluation was finished in 2001. The other is TENDL [2], which is the calculated results by TALYS code with default parameters. After the evaluation of these, some new experimental data were measured and some discrepancies with previous experimental data were observed. Therefore, the purpose of this paper is to obtain new nuclear data for $\mathrm{n}+{ }^{239,240,242,244} \mathrm{Pu}$ reactions below $200 \mathrm{MeV}$.

In Sect. 2, the theoretical models are introduced briefly, and in Sect. 3 the calculated results are provided. Finally, a summary is given in Sect. 4 .

\section{Theoretical model}

The theoretical models are outlined briefly here, the detail can be found in Refs. [3, 4]. The optical model is applied to obtain the total, nonelastic, elastic cross sections

\footnotetext{
*e-mail: guo_hairui@iapcm.ac.cn

**e-mail: hanyl@ciae.ac.cn
}

and elastic scattering angular distributions, transmission coefficients and inverse cross sections. Our global phenomenological Woods-Saxon type optical potentials for nucleon [5], deuteron [6], triton [7], helium [8] and alpha [9] at incident energies up to $200 \mathrm{MeV}$ are used. The distorted wave Born approximation theory is applied to calculate the direct inelastic scattering cross sections and angular distributions. The Hauser-Feshbach model with width fluctuation correction and evaporation model are used to analyze the particle emissions from the equilibrium decay processes for the incident energy below and above $20 \mathrm{MeV}$, respectively. The angular-momentum dependent exciton model is applied to calculate the particle emissions from the pre-equilibrium process, and the improved Iwamoto-Harada pick-up model is included in the exciton model for the calculation of light composite particle emissions. The intranuclear cascade emissions of one to four nucleons are considered and calculated with the empirical formula [10]. Fission is considered as a compondnucleus decay channel. The Bohr-Wheeler theory and the Madland-Nix formula are used to calculate the fission rate and fission spectra, respectively.

The nuclear theoretical model code, UNF [11], is used for the incident energy below $20 \mathrm{MeV}$. The double differential cross sections are calculated with the linear momentum dependent exciton state density model. The recoil effects are taken into account strictly, so the energy balance is held in every channels. MEND code [12] is used for $20 \leq E_{n} \leq 200 \mathrm{MeV}$, and the double differential cross sections of emitted particles are given by using the Kalbach systematics. 


\section{Results and analysis}

All cross sections, angular distributions, energy-angle distributions of neutron, proton, deuteron, triton, helium-3 and alpha emissions, number of neutrons per fission, and prompt fission neutron spectra are calculated. Some of the results are shown in the following figures.

The calculated total cross section of $n+{ }^{239} \mathrm{Pu}$ reaction is compared with experimental data [13-16] and evaluated data from JENDL-4/HE and TENDL in Fig. 1. The present result reproduces the experimental data rather well and differs slightly from those in JENDL-4/HE and TENDL. It should be mentioned that all resonance cross sections in this work are taken from JENDL-4.0.

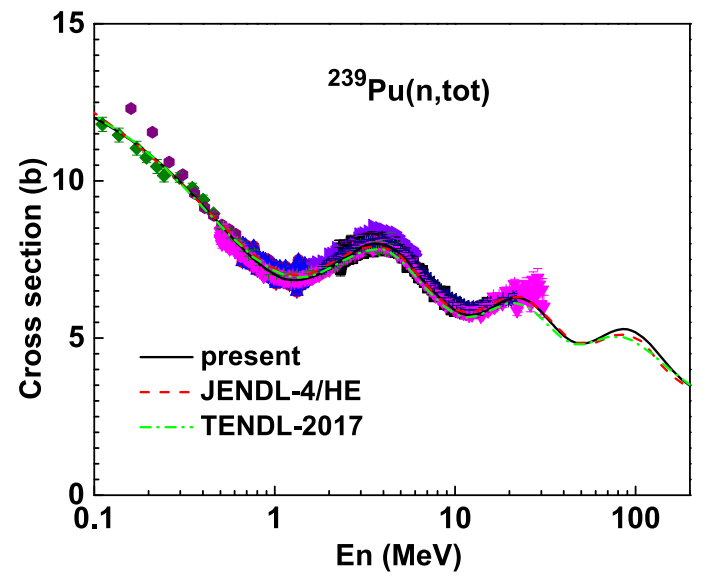

Figure 1. Calculated total cross section (solid line) for $n+{ }^{239} \mathrm{Pu}$ reaction compared with the experimental data and the evaluated data from JENDL-4/HE (dashed line) and TENDL (dash-dotted line).

The fission cross section for $\mathrm{n}+{ }^{239} \mathrm{Pu}$ reaction is given in Fig. 2. The calculated result is in good agreement with the experimental data [17-19]. It can be seen that the data from TENDL are larger than the experimental data for $40 \leq$ $E_{n} \leq 120 \mathrm{MeV}$.

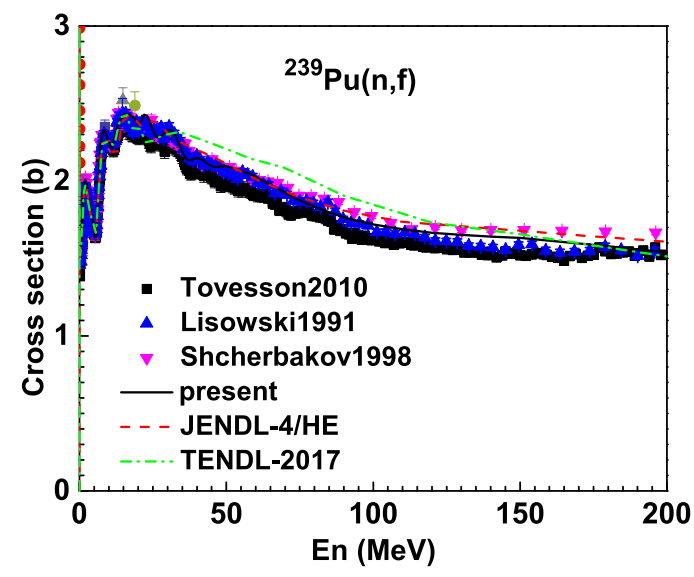

Figure 2. Same as Fig. 1, but for fission cross section.

The fission cross section for $\mathrm{n}+{ }^{240} \mathrm{Pu}$ reaction is given in Fig. 3. The calculated result is in good agreement with the recent experimental data [20, 21]. It can be seen that the present result is a little larger than the data from JENDL-4/HE below $20 \mathrm{MeV}$ where the present result fits the recent experimental data from Refs. [20, 21] better, while the data from JENDL-4/HE fit the experimental data from Refs. [22, 23] better.
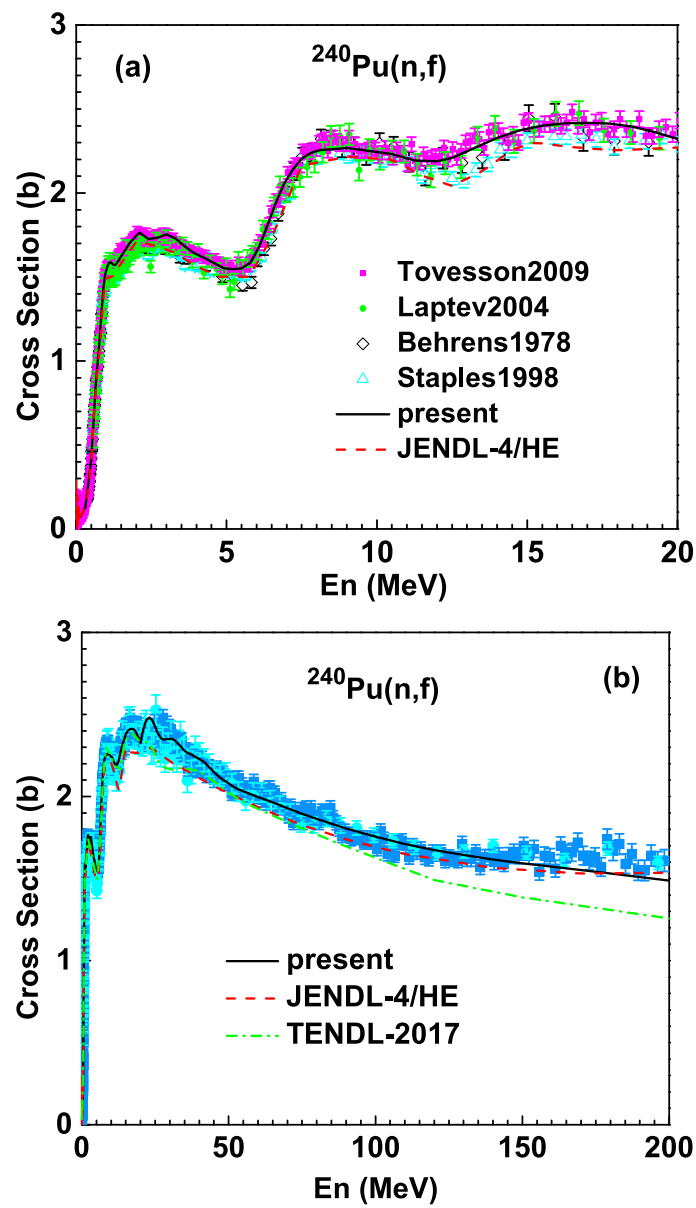

Figure 3. Calculated result of fission cross section (solid lines) for $\mathrm{n}+{ }^{240} \mathrm{Pu}$ reaction compared with the experimental data and the evaluated data from JENDL-4/HE (dashed lines) and TENDL (dash-dotted line). (a) at incident energies below $20 \mathrm{MeV}$, (b) at incident energies below $200 \mathrm{MeV}$.

The $(\mathrm{n}, \gamma)$ reaction cross section for $\mathrm{n}+{ }^{242} \mathrm{Pu}$ reaction is given in Fig. 4. The present result reproduces the new experimental data from Ref. [24] well, while the data from JENDL-4/HE pass through the old experimental data from Ref. [25]. The new experimental data measured at LANSCE are about 3 times lower than the old ones. Therefore, more investigation into the experimental data is needed.

The fission cross section for $\mathrm{n}+{ }^{242} \mathrm{Pu}$ reaction is given in Fig. 5. The present result is in good agreement with the recent experimental data from Ref. [20] over the entire energy region, while the data from JENDL-4/HE is consistent with the experimental data from Ref. [23]. TENDL does not reproduce the experimental data at high energies.

The fission cross section for $\mathrm{n}+{ }^{244} \mathrm{Pu}$ reaction is given in Fig. 6. The calculated result is in good agreement with 


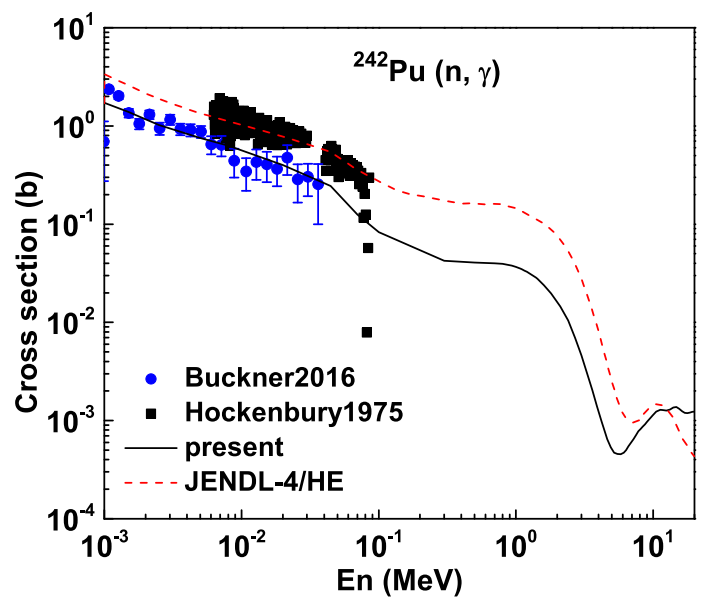

Figure 4. Calculated result of ${ }^{242} \mathrm{Pu}(\mathrm{n}, \gamma)$ reaction cross section (solid line) compared with the experimental data and the evaluated data from JENDL-4/HE (dashed line).

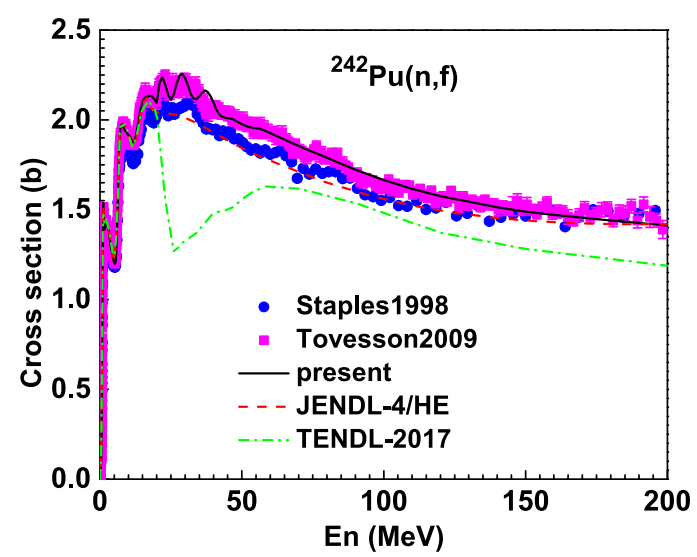

Figure 5. Calculated result of fission cross section (solid line) for $\mathrm{n}+{ }^{242} \mathrm{Pu}$ reaction compared with the experimental data and the evaluated data from JENDL-4/HE (dashed line) and TENDL (dash-dotted line).

the experimental data [23]. There is no evaluated data for this reaction in JENDL-4/HE.

The variation tendency of reaction cross sections related to the target mass numbers is very important for the prediction of nuclear data on neutron-actinides reactions. The trend of fission cross sections for $\mathrm{n}+{ }^{240,242,244} \mathrm{Pu}$ reactions are shown in Fig. 7. The fission cross sections decrease as the mass number increases.

\section{Summary}

The nuclear data on $\mathrm{n}+{ }^{239,240,242,244} \mathrm{Pu}$ reactions are obtained in the incident energy region below $200 \mathrm{MeV}$. Some new experimental data are taken into consideration. The present results are in good agreement with the experimental data generally, and reproduce them better than the evaluated data from JENDL-4/HE and TENDL for some reaction channels. The present results have been transformed into ENDF-B formatted data files for application.

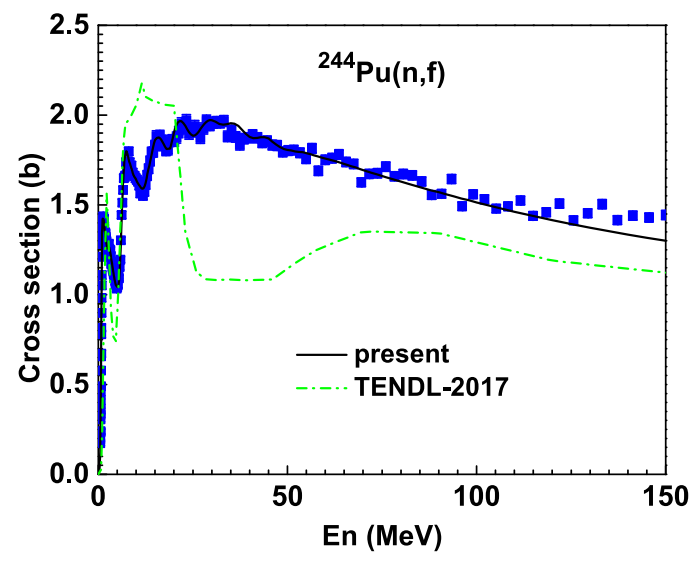

Figure 6. Calculated result of fission cross section (solid line) for $\mathrm{n}+{ }^{244} \mathrm{Pu}$ reaction compared with the experimental data and the evaluated data from TENDL (dash-dotted line).

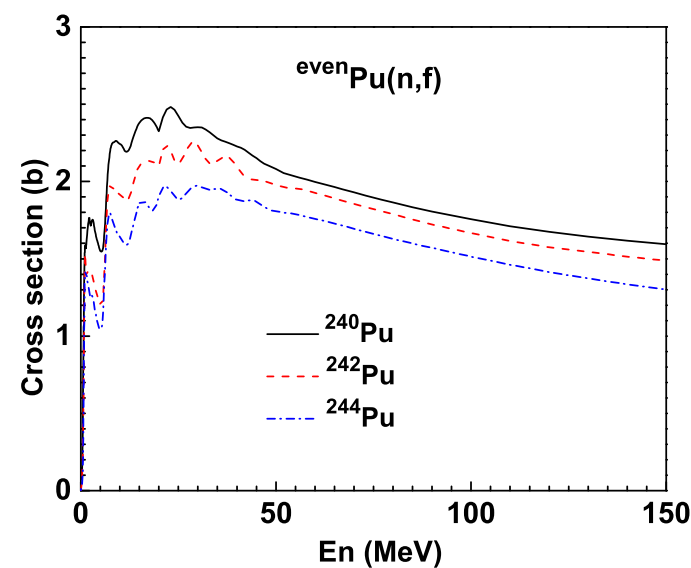

Figure 7. Calculated results of fission cross sections for ${ }^{240} \mathrm{Pu}$ (solid line), ${ }^{242} \mathrm{Pu}$ (dashed line), and ${ }^{244} \mathrm{Pu}$ (dash-dotted line).

\section{Acknowledgments}

This work is supported by China National Natural Science Foundation-NSAF under Grant No.U1630122 and NPL, CAEP under Project 2019BA01. This work is also a part of IAEA Coordinated Research Projects (CRPs) on Recommended Input Parameter Library (RIPL) for Fission Cross Section Calculations under Contract No. 20464.

\section{References}

[1] S. Kunieda et al., JAEA-Conf 2016-004, p. 41-46 (2016).

[2] D. Rochman, A.J. Koning, J.Ch. Sublet, M. Fleming, et al, proceedings of the International Conference on Nuclear Data for Science and Technology, September 11-16, 2016, Bruges, Belgium.

[3] Hairui Guo, Yinlu Han, Tao Ye et al., Annals of Nuclear Energy 108, 151-162 (2017).

[4] Hairui Guo, Yinlu Han, Chonghai Cai, Nucl. Sci. Tech. 30, 13 (2019).

[5] Yinlu Han, Yongli Xu, Haiying Liang et al., Phys. Rev. C 81, 024616 (2010). 
[6] Yinlu Han, Yuyang Shi, Qingbiao Shen, Phys. Rev. C 74, 044615 (2006).

[7] Yongli Xu, Hairui Guo, Yinlu Han, Qingbiao Shen, Int. J. Mod. Phys. E 24, 1550005 (2015).

[8] Yongli Xu, Hairui Guo, Yinlu Han, Qingbiao Shen, SCIENCE CHINA Physics, Mechanics \& Astronomy 54, 2005 (2011).

[9] Xinwu Su and Yinlu Han, Int. J. Mod. Phys. E 24, 1550092 (2015).

[10] Qingbiao Shen et al., Second Inter. Conf. on Accelerator-Driven Transmutation Technologies and Applications, Kalmar, Sweden, p. 686, 1997.

[11] Jingshang Zhang, Nucl. Sci. Eng. 142, 207-219 (2002).

[12] Chonghai Cai, Nucl. Sci. Eng. 153, 93 (2006).

[13] D.G. Foster, D.W. Glasgow, Phys. Rev. C 3, 576-603 (1971).

[14] W.P. Poenitz, J.F. Whalen, A.B. Smith, Nucl. Sci. Eng. 78, 333-341 (1981).

[15] W.P. Poenitz, J.F. Whalen, ANL-NDM-80 (1983).

[16] A.B. Smith, P. Guenther, J. Whalen, J. Nucl. Energy 27, 317 (1973).
[17] F. Tovesson, T.S. Hill, Nucl. Sci. Eng. 165, 224-231 (2010).

[18] P.W. Lisowski et al., Proc. of the NEANDC Specialists Meeting on Neutron Cross Section Standards for the Energy Region above $20 \mathrm{MeV}, 21-23$ May, 1991, Uppsala, Sweden.

[19] O. Shcherbakov et al., ISINN-6, 306 (1998).

[20] F. Tovesson, T.S. Hill, M. Mocko et al., Phys. Rev. C 79, 014613 (2009).

[21] A. B. Laptev, A.Yu. Donets, V.N. Dushin et al., Proc. Int. Conf. Nuclear Data for Science and Technology, Santa Fe, p. 865-869, 2004.

[22] J. W. Behrens, R. S. Newbury, and J. W. Magana, Nucl. Sci. Eng. 66, 433-441 (1978).

[23] P. Staples and K. Morley, Nucl. Sci. Eng. 129, 149163 (1998).

[24] M. Q. Buckner, C.Y. Wu, R.A. Henderson et al., Phys. Rev. C 93, 044613 (2016).

[25] R. W. Hockenbury, A. J. Sanislo, N. N. Kaushal, Proc. Conf. on Nuclear Cross Sections and Technology, Washington, 2, p. 584, 1975. 